\title{
Old problems are still there: robot intelligence as the interplay between many components of complex architectures
}

\author{
Fulvio Mastrogiovanni · Nak Young Chong
}

(C) Springer-Verlag Berlin Heidelberg 2012

The goal of the two Special Issues about "Artificial Intelligence techniques for Robotics: sensing, representation and action" is to present, discuss and assess recent advances in the use of Artificial Intelligence methods to build better robotic systems.

When we say "better", what are we talking about? Nowadays we know that elephants do not play chess [1] and, despite the astonishing performance of IBM Watson in Jeopardy [2], we argue that robot intelligence is a property of complex robot systems interacting with real world environments (including the robot itself). The question here is how can we achieve a level of robot performance that can, even loosely, resemble what any person can see in such movies as "Robot and Frank" [3]?

As a matter of fact, as we expect robots to leave lab settings and to take part to our everyday life, either at home or in public spaces [4], the concept of AI as a separable and somewhat peculiar part of robot architectures shows its intrinsic limitations. The stress on sensing, representation and action of this Special Issue is motivated by the need of

F. Mastrogiovanni $(\varangle)$

Department of Informatics, Bioengineering, Robotics and Systems Engineering, University of Genoa,

Via Opera Pia 13, 16145 Genoa, Italy

e-mail: fulvio.mastrogiovanni@unige.it

\section{N. Y. Chong}

School of Information Science, Japan Advanced Institute of Science and Technology, 1-1 Asahidai, Nomi, Ishikawa 923-1292, Japan

e-mail: nakyoung@jaist.ac.jp distributing AI principles and methods at various levels of robot architectures, with respect to both hardware and software components.

It becomes evident that, in order to cope with the plethora of situations, events, objects, places and humans the world is full of, robot intelligence "as it is" is rather insufficient, both with respect to the scope of achievable performance and to actual techniques and design tools for designing engineered robot behaviour.

This motivates a broad research agenda in AI techniques for Robotics, involving such different-yet intertwinedresearch areas as software architectures, sensing and perception, intelligent control, behaviour and reasoning models, distributed knowledge representation and computational ontologies, engineering tools and fast-prototyping techniques, learning, real-time systems and robot morphology.

Starting from these different research fields, the need arises to design novel integration paradigms to face complex challenges in real-world service tasks, specifically since robot intelligence is expected to play major roles when interacting with humans and when dealing with everyday situations, like human assistance, housekeeping or autonomous driving, just to name three of the most common scenarios Robotics like to play with.

The reader may have noticed that we talked about two Special Issues. Indeed, what you are reading is the Guest Editorial to the first Special Issue. There is still much work to be done on AI techniques for Robotics. The community recognized this situation by sending many valuable articles discussing the previous topics and worth publication. The second Special Issue is on the way.

The Guest Editors would like to acknowledge the journal Editor-in-Chief, the Administrative and Editorial Staff, all the reviewers and, above all, all the authors for their valuable contributions. 


\section{References}

1. Brooks RA (1990) Elephants don't play chess. In: Robotics and autonomous systems, vol 6. pp 3-15

2. See the official IBM Watson webpage at http://www-03.ibm.com/ innovation/us/watson/
3. See the official motion picture webpage at http://robotandfrank-film. com/

4. See the official Robot Companion for Citizens FET flagship candidate initiative at http://www.robotcompanions.eu/ 